\title{
ULTIMATE THEORETICAL STRENGTH OF CEMENTITE IN THE (100), (010) AND (001) PLANES
}

\author{
L. E. Karkina, A. R. Kuznetsov, I. N. Karkin \\ M.N. Miheev Institute of Metal Physics, Ural Branch of the Russian Academy of Sciences, 18 S. Kovalevskoy St., \\ Ekaterinburg, 620990, Russian Federation \\ *Corresponding author. E-mail: lidiya.karkina@imp.uran.ru; address for correspondence: ul. S. Kovalevskoy, 18, \\ 620990, Ekaterinburg, Russian Federation. Tel.: +7 (343) 3783647; fax: +7 (343) 3745244
}

Atomistic analysis of the ultimate theoretical strength of cementite in the (100), (010) and (001) planes has been performed using the molecular dynamics method. To characterize fracture, the decohesion energy, the Griffith surface energy for crack planes and the brittle fracture parameter in the Rice-Thompson model have been calculated. It is demonstrated that crack blunting may occur only in the (001) plane due to plastic strain relaxation at its top. The fracture parameter is either too large, or plastic relaxation of stresses at the crack tip is impossible in the (010) and (100) planes due to the location geometry of the studied cleavage planes and the easiest modes of plastic relaxation. The crack in the (100) and (010) planes opens in a brittle way.

Keywords: brittle fracture parameter, atomistic modeling, decohesion energy, unstable stacking fault energy, cementite.

DOI: $10.17804 / 2410-9908.2016 .5 .067-076$

\section{References}

1. Shchastlivtsev V.M., Mirzaev D.A., Yakovleva I.L., Okishev K.Yu., Tabatchikova T.I., Khlebnikova Yu.V. Perlit v uglerodistykh stalyakh [Pearlite in Carbon Steels]. Ekaterinburg, UrO RAN Publ., 2006, 312 p. (In Russia).

2. Koreeda A., Shimizu K. Dislocations in cementite. Phil. Mag., 1968, vol. 17, iss. 149, pp. 1083-1086. DOI: 10.1080/14786436808223185.

3. Inoue A., Ogura T., Masumoto T. Deformation and fracture behaviours of cementite. Trans. JIM., 1976, vol. 17, pp. 663-672.

4. Inoue A., Ogura T., Masumoto T. Dislocation structure of cementite in cold-rolled carbon steels. J. Japan Inst. Metals., 1973, vol. 37, no. 8, pp. 875-882.

5. Inoue A., Ogura T., Masumoto T. Microstructures of deformation and fracture of cementite in pearlitic carbon steels strained at various temperatures. Met. Trans., 1977, vol. 8A, pp. 1689-1695.

6. Nishiyama Z., Kore'eda A., Katagiri S. Study of plane defects in the cementite by transmission electron microscopy. Trans. JIM., 1964, vol. 5, pp. 115-121.

7. Rice J.R., Thompson R. Ductile versus brittle behaviour of crystals. Phil. Mag., 1974, vol. 29, iss. 1, pp. 73-97. - DOI: 10.1080/14786437408213555.

8. Bitzek E., Kermode J.R., Gumbsch P. Atomistic aspects of fracture. Int. J. Fracture, 2015, vol. 191, iss. 1, pp. 13-30. - DOI: 10.1007/s10704-015-9988-2.

9. Terentyev D., He X. Properties of grain boundaries in BCC iron and iron-based alloys. An atomistic study. Open Report of the Belgian Nuclear Research Centre, SCK•CEN-BLG-1072, 2010, 70 p. ISSN 1379-2407.

10. Starikov S.A., Kuznetsov A.R., Karkina L.E., Sagaradze V.V. Ultimate theoretical strength of fcc Fe-Ni alloy polycrystals. Diagnostics, Resource and Mechanics of materials and structures, 2015, iss. 6, pp. 58-62. DOI: 10.17804/2410-9908.2015.6.058-062. Available at: http://dreamjournal.org. 
11. Sun Y., Rice J.R., Truskinovsky L. Dislocation Nucleation Versus Cleavage in $\mathrm{Ni}_{3} \mathrm{AI}$ and Ni. Mat. Res. Soc. Symp. Proc., 1991, vol. 213, pp. 243-248. DOI: 10.1557/PROC-213-243.

12. Kelly A., Tyson W., Cottrell A.H. Ductile and brittle crystals. Phil. Mag., 1967, vol. 15, iss. 135, pp. 567-586. DOI: 10.1080/14786436708220903.

13. Rosato V. Comparative behavior of carbon in bec and fec iron. Acta Metall., 1989, vol. 37, iss. 10, pp. 2759-2763. DOI: 10.1016/0001-6160(89)90310-6.

14. Daw M.S., Baskes M.I. Embedded atom method: derivation and application to impurities, surfaces and other defects in metals. Phys. Rev., 1984, vol. 29B, no. 12, pp. 6443-6453. DOI: 10.1103/PhysRevB.29.6443.

15. Johnson R.A., Dienes G.J., Damask A.C. Calculation of the energy and migration characteristics of carbon and nitrogen in $\alpha$-iron and vanadium. Acta Metall., 1964, vol. 12, iss. 11, pp. 1215-1224. DOI: 10.1016/0001-6160(64)90105-1.

16. Levchenko E.V., Evteev A.V., Belova I.V., Murch G.E. Molecular dynamics simulation and theoretical analysis of carbon diffusion in cementite. Acta Mater., 2009, vol. 57, iss. 3, pp. 846-853. DOI: 10.1016/j.actamat.2008.10.025.

17. Kar'kina L.E., Kar'kin I.N., Kuznetsov A.R Atomistic simulation of stacking faults in (001), (010), and (100) planes of cementite. Physics of Metals and Metallography, 2014, vol. 115, iss. 1, pp. 85-97. DOI: 10.1134/S0031918X14010086.

18. Kar'kina L.E., Kar'kin I.N., Zubkova T.A. Atomistic simulation of stacking faults in cementite: Planes containing vector [100]. Physics of Metals and Metallography, 2014, vol. 115, iss. 8, pp. 814-829. DOI: 10.1134/S0031918X14080067.

19. Kar'kina L.E., Kar'kin I.N. Atomistic simulation of stacking faults in cementite: Planes containing vector [010]. Physics of Metals and Metallography, 2014, vol. 115, iss. 8, pp. 830-842. DOI: 10.1134/S0031918X14080079.

20. Kar'kina L.E., Zubkova T.A., Yakovleva I.L. Dislocation structure of cementite in granular pearlite after cold plastic deformation. Physics of Metals and Metallography, 2013, vol. 114, iss. 3, pp. 234-241. DOI: 10.1134/S0031918X13030095. 
Подана в журнал: 20.10 .2016

УДК 669.14:539.4

DOI: $10.17804 / 2410-9908.2016 .5 .067-076$

\title{
ПРЕДЕЛЬНАЯ ТЕОРЕТИЧЕСКАЯ ПРОЧНОСТЬ ЦЕМЕНТИТА В ПЛОСКОСТЯХ (100), (010) и (001)
}

\author{
Л. Е. Карькина ${ }^{*}$, А. Р. Кузнецов, И. Н. Карькин \\ Федеральное государственное бюджетное учреждение науки Институт физики металлов \\ имени М.Н. Михеева Уральского отделения Российской академии наук, ул. С. Ковалевской, 18, 620990, \\ Екатеринбург, Российская Федерачия \\ *Ответственный автор. Электронная почта: lidiya.karkina@imp.uran.ru; aдpec для переписки: 620990, \\ ул. С. Ковалевской, 18, Екатеринбург, Российская Федерация. Телефон: +7 (343) 378-36-47; \\ факс: +7 (343) 374-52-44
}

Проведен атомистический анализ предельной теоретической прочности цементита в плоскостях (100), (010) и (001) с использованием метода молекулярной динамики. Для характеристики разрушения рассчитана энергия декогезии, поверхностная энергия Гриффитса для плоскостей раскрытия трещин и параметр хрупкого разрушения в модели РайсаТомпсона. Показано, что только в плоскости (001) возможно затупление образующейся трещины вследствие пластической релаксации напряжений в ее вершине. В плоскостях (010) и (100) из-за геометрии расположения изученных плоскостей скола и наиболее легких мод пластической релаксации параметр разрушения или слишком велик, или вообще невозможна пластическая релаксация напряжений в вершине трещины, трещина в плоскостях (100) и (010) раскрывается хрупко.

Ключевые слова: параметр хрупкого разрушения, атомистическое моделирование, энергия декогезии, энергия нестабильного дефекта упаковки, цементит.

\section{1. Введение}

Высокие прочностные свойства сталей перлитного класса обусловлены выделениями пластинчатого или глобулярного цементита в феррите [1]. В настоящее время проведен большой объем экспериментальных и теоретических исследований механических свойств перлитных сталей. В [2-4] с использованием оптической, сканирующей и электронной просвечивающей микроскопии установлено, что цементит может деформироваться не только при повышенных температурах, но и при комнатной. В меньшей степени экспериментально изучены механизмы разрушения цементита. Так, в [5] с использованием просвечивающей электронной микроскопии показано, что при температуре менее $-78{ }^{\circ} \mathrm{C}$ разрушение цементита происходило сколом по таким кристаллографическим плоскостям как (110), (100) или (210). При комнатной температуре в $[5,6]$ установлено, что деформация и разрушение происходят по плоскостям (100) и (010).

Способность трещины к нормальному раскрытию характеризуется как значением поверхностной энергии Гриффитса, так и склонностью данной трещины к затуплению путем формирования пластической зоны в ее вершине. Количественная характеристика меры хрупкого поведения материала может быть учтена в модели Райса-Томпсона [7], которая учитывает значения поверхностной энергии Гриффитса, склонности материала к пластической релаксации, а также геометрические факторы, учитывающие соотношение между плоскостями скола и системами скольжения.

Макроскопические процессы разрушения практически полностью определяются процессами, происходящими на атомном уровне [8]. Моделированию разрушения в ОЦК-железе и сплавах на его основе уделялось большое внимание в последнее время $[8,9]$. ГЦК железо и 
сплавы на его основе тоже начали изучаться на атомном уровне [10]. В работе на атомном уровне рассмотрены процессы разрушения в цементите.

\section{2. Постановка задачи и методика моделирования}

Количественная характеристика меры хрупкого поведения цементита оценивалась в рамках модели Райса-Томпсона [7]. В этой модели мера хрупкости определяется двумя энергетическими величинами: энергией нестабильного дефекта упаковки (ДУ) и энергией декогезии. Эти характеристики вычислялись с использованием метода молекулярной динамики для (001), (010) и (100) плоскостей цементита. В модели Райса-Томпсона рассматривается трещина нормального отрыва I типа в присутствии растягивающих напряжений, перпендикулярных плоскости трещины. Предложенный в [11] безразмерный параметр q, характеризующий меру хрупкости, имеет вид:

$$
q=G_{d} / G_{c}=\frac{\gamma_{u s}\{1+(1-v)] \operatorname{tg}^{2} \varphi}{2 \gamma_{c} f^{2}(\theta)},
$$

где $G_{\mathrm{c}}=2 \gamma_{\mathrm{c}}-$ энергия декогезии; $\gamma_{\text {us }}-$ энергия нестабильного ДУ; $v$ - коэффициент Пуассона;

$$
G_{d}=\frac{\gamma_{u s}\{1+(1-v)] \operatorname{tg}^{2} \varphi}{f^{2}(\theta)}
$$

- условие зарождения дислокаций для трещины I типа.

На рис.1 схематически представлена геометрия трещины с нормалью к плоскости трещины вдоль ОУ.

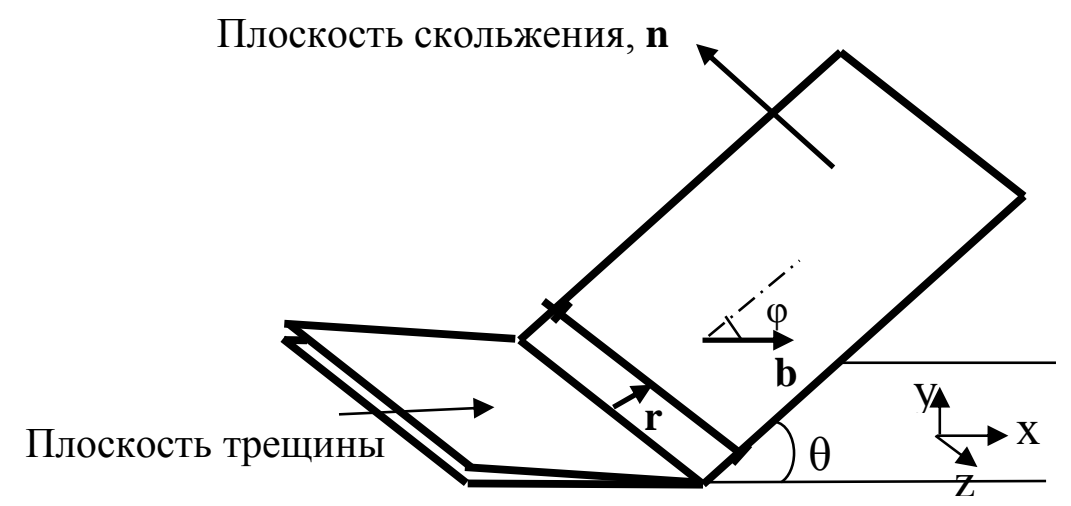

Рис. 1. Схематическое изображение трещины нормального отрыва:

OY - нормаль к плоскости раскрывающейся трещины; $\mathbf{n}$ - нормаль к плоскости скольжения

дислокаций с вектором Бюргерса $\mathbf{b}$, которые способствуют релаксации напряжений в вершине трещины

Фронт трещины совпадает с OZ, плоскость скольжения имеет нормаль $\mathbf{n} ; \theta$ - угол между плоскостью трещины и плоскостью скольжения дислокации; $\varphi$ - угол между вектором Бюргерса и нормалью к линии пересечения плоскости скольжения дислокации и плоскости трещины; r лежит в плоскости скольжения перпендикулярно к фронту трещины OZ:

$$
f(\theta)=\cos ^{2} \frac{\theta}{2} \sin \frac{\theta}{2} \text {. }
$$


Значение q вычислялось для каждой из выбранных типов плоскостей скола и возможных систем скольжения дислокаций в цементите с наименьшим значением $\gamma_{\text {us }}$.

При вычислении энергии декогезии верхняя половина кристаллита сдвигалась вдоль нормали к выбранной плоскости до образования двух свободных поверхностей. При опреде-

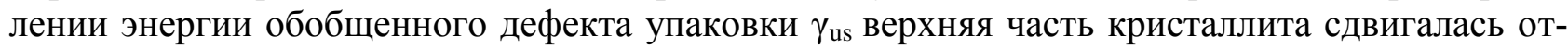
носительно нижней в рассматриваемой плоскости. Вектор смещения $\boldsymbol{f}$ увеличивался с шагом до значения, совпадающего с трансляцией решетки вдоль выбранного направления. Из графика зависимости энергии поверхностного дефекта от величины сдвига, параллельного вектору Бюргерса полных или частичных дислокаций, осуществляющих пластическую деформацию, можно получить значение энергии нестабильных дефектов упаковки $\gamma_{\text {us }}$ (максимальное значение энергии ДУ вдоль выбранного направления сдвига). Параметр был введен в теории разрушения [12] и использовался при оценке склонности изучаемой трещины к пластической релаксации. Чем меньше этот параметр, тем легче осуществляется движение дис-


решетки при движении рассматриваемой дислокации.

При расчетах методом молекулярной динамики (МД) был использован потенциал [13]. Взаимодействие $\mathrm{Fe}-\mathrm{Fe}$ задается N-частичным ЕАМ-потенциалом, построенным в схеме «погруженного атома» [14]. Для описания взаимодействия пар атомов $\mathrm{Fe}-\mathrm{C}$ и $\mathrm{C}-\mathrm{C}$ использован парный потенциал Джонсона [15], обсуждение возможности использования потенциала даны в [16]. Выбранный потенциал межатомного взаимодействия дает минимум энергии решетки цементита с параметрами решетки $a=4,393 \AA ; b=5,074 \AA ; c=6,345 \AA$, которые совпадают с их экспериментальными значениями в пределах 1-6\%.

\section{3. Результаты и обсуждение}

\section{1. Энергия декогезии для различных сечений в плоскостях (001), (010) и (100) цементита}

На рис.2 показана элементарная ячейка цементита в проекции на плоскости (001), (010) и (100) цементита [17].

Для различных «кубических» плоскостей цементита существует от двух до четырех неэквивалентных сечений, различающихся энергией и заполнением атомами $\mathrm{Fe}$ и $\mathrm{C}$ ближайших плоскостей. В [17] показано, что для плоскости (001) существует 2 различных сечения (I и II), для плоскости (100) - 3 сечения, для плоскости (010) - 4 различных сечения. Для каждого из сечений в этих плоскостях была вычислена энергия декогезии и энергия поверхности, образующейся при раскрытии трещины нормального отрыва. На рис. 3 представлена энергия декогезии $G_{\text {c }}$ в зависимости от расстояния между атомными плоскостями $U$ для плоскости (001) (a), (100) (б) и (010) (в). В табл. 1 суммированы значения энергии декогезии и энергии поверхностных дефектов $\gamma_{\mathrm{c}}=G_{\mathrm{c}} / 2$, упорядоченные в порядке возрастания этих величин. Чем ниже значение энергии декогезии (при прочих равных условиях), тем легче происходит образование трещины.

Таблица 1. Энергия декогезии $G_{c}$ и энергия поверхностных дефектов $\gamma_{c}$ для рассмотренных плоскостей раскрытия трещин

\begin{tabular}{|c|c|c|c|c|c|}
\hline $\begin{array}{l}\text { Плоскость } \\
\text { трещины }\end{array}$ & $G_{\mathrm{c}}$, Дж/ $\mathrm{m}^{2}$ & 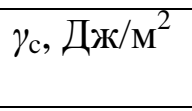 & $\begin{array}{l}\text { Плоскость } \\
\text { трещины }\end{array}$ & $G_{\mathrm{c}}$, Дж/ $\mathrm{m}^{2}$ & 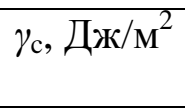 \\
\hline (100) III & 3,638 & 1,819 & (100) II & 4,515 & 2,257 \\
\hline (001) II & 3,668 & 1,834 & (010) II & 4,617 & 2,308 \\
\hline (100) I & 3,876 & 1,938 & (010) IV & 4,680 & 2,340 \\
\hline (001) I & 4,095 & 2,047 & (010) III & 4,818 & 2,409 \\
\hline$(010) \mathrm{I}$ & 4,273 & 2,136 & & & \\
\hline
\end{tabular}


Самые низкие значения поверхностной энергии на разрыв $\gamma_{\mathrm{c}} \sim 1,8$ Дж/м ${ }^{2}$ получены для плоскостей (100) III типа и (001) II типа. В этих плоскостях раскрытие трещины может происходить легче, чем в других плоскостях. Самые высокие значения $\gamma_{\mathrm{c}} \sim 2,2$ Дж/м² получены для различных сечений плоскости (010), табл. 1. В этих плоскостях процесс раскрытия трещин должен осуществляться наиболее трудно.



$\mathrm{d}_{1}=1,173 \AA, \mathrm{d}_{2}=0,825 \AA$

$a$



$\mathrm{d}_{1}=0,426 \AA, \mathrm{d}_{2}=0,729 \AA, \mathrm{d}_{3}=0,615 \AA$

б

(001)



$\mathrm{d}_{1}=0,457 \AA, \mathrm{d}_{2}=0,228 \AA, \mathrm{d}_{3}=0,760 \AA, \mathrm{d}_{4}=0,406 \AA$

B

Рис. 2. Элементарная ячейка $\mathrm{Fe}_{3} \mathrm{C}$ в проекции на различные «кубические» плоскости цементита. Крупными символами обозначены атомы Fe, мелкими символами - атомы $\mathrm{C}$ на различных уровнях плоскостей (001) (a), плоскостей (100) (б) и плоскостей (010) (в) 


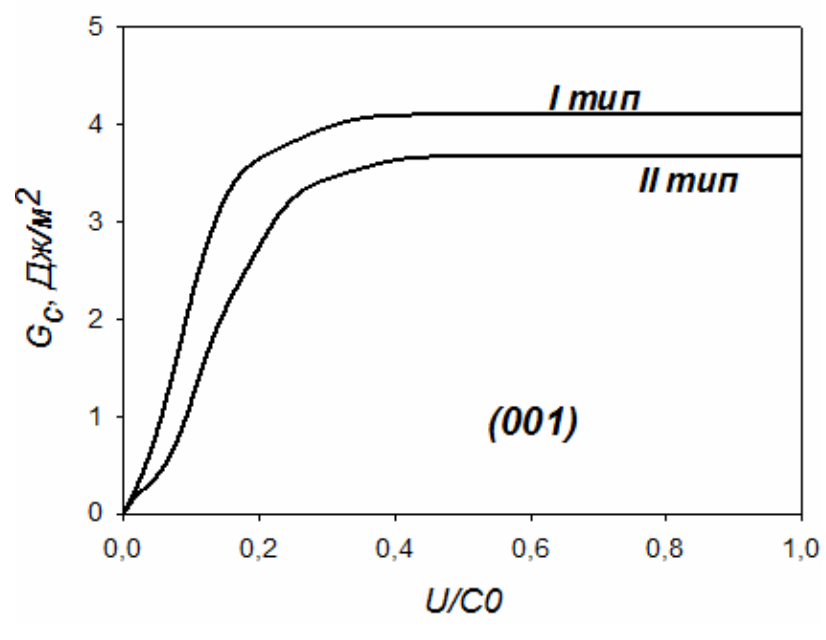

$a$

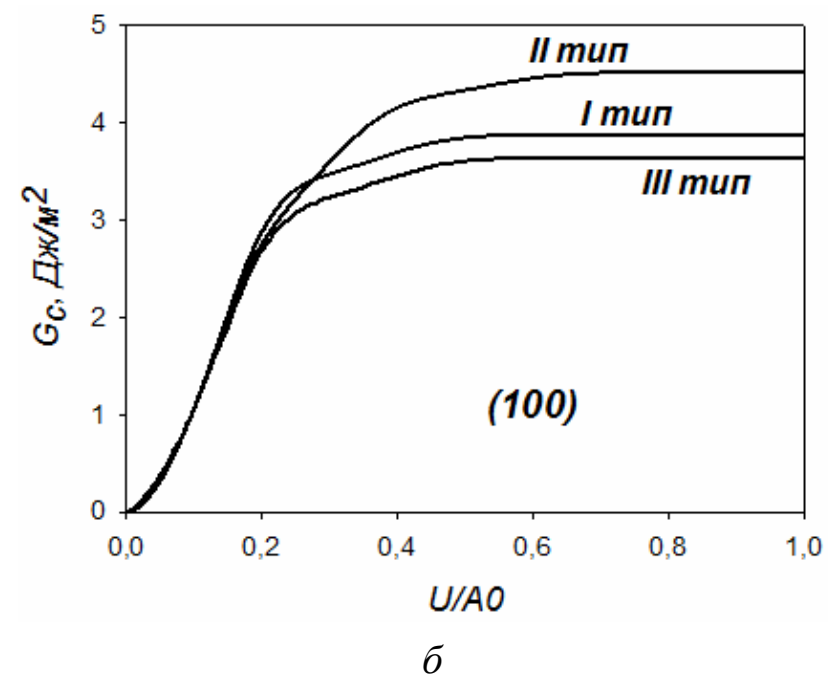

$\sigma$

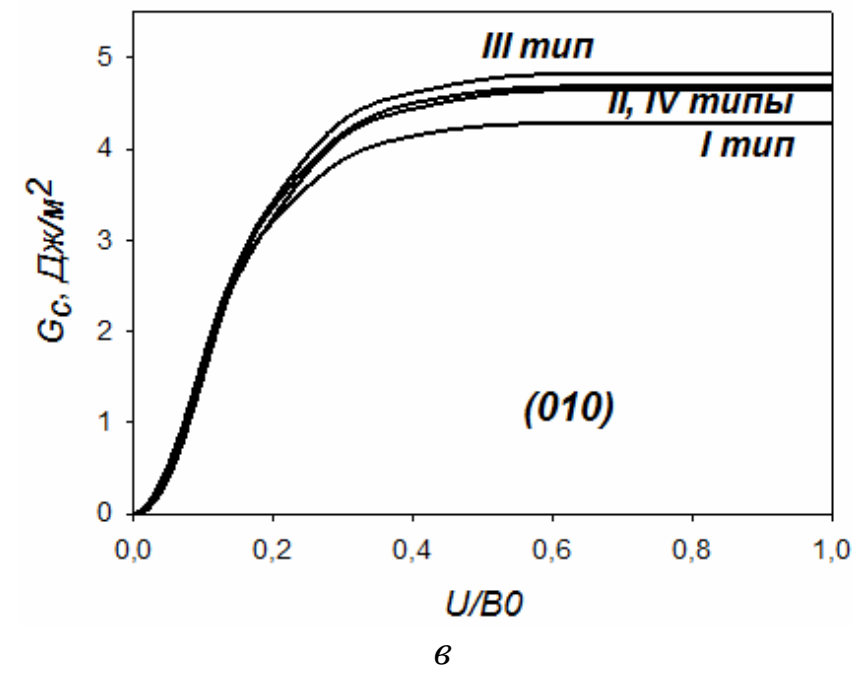

Рис. 3. Зависимость энергии декогкзии $G_{\mathrm{c}}$ от расстояния между атомными плоскостями для плоскостей (001) (a), (100) (б) и (010) (в) цементита

\section{2. Энергия нестабильных дефектов упаковки}

Релаксационные характеристики трещин оценивались по величине энергии нестабильных дефектов упаковки $\gamma_{\text {us }}$, характеризующих способность к скольжению при сдвиге одной части кристалла относительно другой. Результаты расчетов энергии обобщенных дефектов упаковки в плоскостях, содержащих векторы Бюргерса [100] и [010] цементита представлены в работах $[18,19]$. Наиболее легкое скольжение получено для движения частичных дислокаций с вектором Бюргерса $\alpha[010]$, где $\alpha \sim 0.4$, в плоскости (001) цементита. Именно эта мода деформации наблюдалась экспериментально при небольшой степени деформации в карбидах глобулярного перлита [20].

Таблица 2 - Энергии нестабильных ДУ $\left(\gamma_{\mathrm{us}}, \mathrm{J} / \mathrm{m}^{2}\right)$, соответствующие полным и частичным сдвигам в сечениях I и II плоскости (001), плоскостей (011) и (103) цементита

\begin{tabular}{|c|c|c|c|}
\hline Плоскость & $\begin{array}{c}\text { Вектор Бюргерса частичных } \\
\text { дислокаций } \\
\end{array}$ & 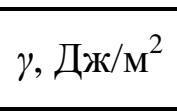 & $\gamma_{u s}$, Дж/ $\mathrm{M}^{2}$ \\
\hline (001) I тип & $\sim 0,4[010]$ & 0,59 & 2,21 \\
\hline (001) II тип & $\sim 0,4[010]$ & 0,46 & 2,91 \\
\hline (011) I тип & $\sim 0,1[1-33]$ & 0,31 & 2,82 \\
\hline (103) I тип & $\sim 0,8[-301]$ & 0,36 & 2,83 \\
\hline
\end{tabular}


В табл. 2 (1 строка) даны значения энергии ДУ в точке минимума, соответствующего сдвигу $\alpha[010]$, и значения энергии нестабильного ДУ, характеризующего легкость образования этого дефекта. Движение полной дислокации характеризуется значительно более высоким значением $\gamma_{\text {us. }}$.

В работах [17-19] были сопоставлены значения энергий стабильных и нестабильных ДУ цементита с другими металлами. Значения энергий стабильных ДУ имеют значения сопоставимые с другими материалами. Однако для цементита даже самые низкие значения $\gamma_{u s}$ в несколько раз превышают значения, полученные для чистых металлов и упорядоченных сплавов. Высокие значения $\gamma_{u s}$ препятствуют формированию пластической зоны в цементите. Действительно, экспериментально установлено, что в зернистом перлите деформация цементита начинается только после общей деформации на степени $~(10-20) \%$, когда возрастают внутренние напряжения, обусловленные скоплениями дислокаций на межфазной границе феррит-цементит. В табл. 2 во 2-й и 3-й строках добавлены только те моды деформации, которые имеют значения $\gamma_{u s}$, близкие к значениям для плоскости (001). Данные, приведенные в табл. 2, получены с использованием МД-моделирования [17-20]. Представленные в табл. 2 моды деформации - это движение частичных дислокаций, ограничивающих дефект упаковки, который имеет достаточно низкое значение энергии.

\section{3. Вычисление параметра разрушения}

Безразмерный параметр $q$ (формулы 1-3), характеризующий меру хрупкости, вычислялся для одной из выбранных плоскостей скола, представленных в табл. 1, и возможных систем скольжения частичных дислокаций, представленных в табл. 2. Для всех рассмотренных плоскостей скола релаксация напряжений в плоскости (001) с наиболее низкими значениями $\gamma_{\text {us }}$ (табл. 2) оказалась невозможной. Для плоскости скола (001) угол $\theta=0$ и $f(\theta)=0$ (3). Для плоскости скола (010) угол $\varphi=\pi / 2$ и релаксация напряжений в плоскости (001) невозможна. Для плоскости скола (100) вектор Бюргерса частичной дислокации 0,4[010] лежит вдоль линии пересечения плоскости скола и плоскости скольжения (001), так что $\varphi=0$ и релаксация напряжений невозможна. Аналогичная ситуация наблюдается для плоскости скола (100). Для плоскости скольжения (011) угол $\theta=0$, для плоскости скольжения (103) угол $\varphi=0$. Для плоскости скола (010) угол $\varphi=0$ для плоскости скольжения (103). Таким образом, для исследованных «кубических» плоскостей скола релаксация напряжений в вершине возникающей трещины возможна только в трех системах скольжения, представленных в табл. 3. Видна существенная разница в значениях $q$. Для системы скольжения [1-33](011) в 1-й и 3-й строках табл. 3 угол $\varphi$ близок к $90^{\circ}$, что дает высокое значение $\operatorname{tg}^{2} \varphi$ в формуле (2) и, соответственно, высокое значение параметра $q$.

В статье [11] показано, что уровень $q_{\mathrm{c}} \sim 0,7-0,9$ является количественной мерой, разделяющей в предложенной схеме хрупкие и пластичные материалы. Значения $q<q_{\text {c }}$ соответствуют пластичным материалам, которые характеризуются высоким значением поверхностной энергии и низким значением сопротивлением сдвигу. Для $q>q_{\mathrm{c}}$ материал является хрупким. Из табл. 3 следует, что единственной плоскостью, в которой возможна релаксация напряжений в вершине распространяющейся трещины и соответственно ее торможение, является плоскость (001). Во всех остальных случаях геометрия взаимного расположения плоскостей скола и систем скольжения препятствует образованию зоны пластической релаксации в вершине трещины, таким образом, трещины в плоскостях (010) и (100) раскрываются хрупко. Именно эти плоскости скола наблюдаются экспериментально [4-6]. 
Таблица 3 - Значения параметра разрушения $q$ для «кубических» плоскостей раскрытия трещин и систем скольжения частичных дислокаций в цементите

\begin{tabular}{|c|c|c|}
\hline Плоскость трещины & Система скольжения & $q$ \\
\hline$(001) \mathrm{I}$ & {$[1-33](011)$} & 25,05 \\
$(001) \mathrm{I}$ & {$[30-1](103)$} & 0,53 \\
$(001) \mathrm{II}$ & {$[30-1](103)$} & 0,59 \\
\hline$(010) \mathrm{IV}$ & {$[1-33](011)$} & 24,43 \\
\hline
\end{tabular}

\section{4. Заключение}

Исследование природы предельной теоретической прочности цементита в модели Райса-Томпсона показало, что только в плоскости (001) возможно затупление образующейся трещины вследствие пластической релаксации напряжений в ее вершине. В плоскостях (010) и (100) из-за геометрии расположения изученных плоскостей скола и наиболее легких мод пластической деформации параметр разрушения $q$ или слишком велик, или вообще невозможна пластическая релаксация напряжений в вершине трещины. Трещина в плоскостях (100) и (010) раскрывается хрупко.

\section{Благодарность}

Работа выполнена в рамках государственного задания ФАНО России (темы «Структура» № 01201463331 и «Деформация» № 01201463327) и комплексной программы УрО РАН (проект № 15-9-12-45). При проведении работы был использован суперкомпьютер «Уран» ИММ УрО РАН.

\section{Литература}

1. Перлит в углеродистых сталях / В. М. Счастливцев, Д. А. Мирзаев, И. Л. Яковлева, К. Ю. Окишев, Т. И. Табатчикова, Ю. В. Хлебникова. - Екатеринбург : УрО РАН, 2006. 312 c.

2. Koreeda A., Shimizu K. Dislocations in cementite // Phil. Mag. - 1968. - Vol. 17, iss. 149. P. 1083-1086. - DOI: 10.1080/14786436808223185.

3. Inoue A., Ogura T., Masumoto T. Deformation and fracture behaviours of cementite // Trans. JIM. - 1976. - Vol. 17. - P. 663-672.

4. Inoue A., Ogura T., Masumoto T. Dislocation structure of cementite in cold-rolled carbon steels // J. Japan Inst. Metals. - 1973. - Vol. 37, no. 8. - P. 875-882.

5. Inoue A., Ogura T., Masumoto T. Microstructures of deformation and fracture of cementite in pearlitic carbon steels strained at various temperatures // Met. Trans. - 1977. - Vol. 8A. P. 1689-1695.

6. Nishiyama Z., Kore'eda A., Katagiri S. Study of plane defects in the cementite by transmission electron microscopy // Trans. JIM. - 1964. - Vol. 5. - P. 115-121.

7. Rice J. R., Thompson R. Ductile versus brittle behaviour of crystals // Phil. Mag. - 1974. Vol. 29, iss. 1. - P. 73-97. - DOI: 10.1080/14786437408213555.

8. Bitzek E., Kermode J. R., Gumbsch P. Atomistic aspects of fracture // Int. J. Fracture. 2015. - Vol. 191, iss. 1. - P. 13-30. - DOI: 10.1007/s10704-015-9988-2.

9. Terentyev D., He X. Properties of grain boundaries in BCC iron and iron-based alloys. An atomistic study // Open Report of the Belgian Nuclear Research Centre. - SCK •CEN-BLG-1072, 2010. - 70 p. - ISSN 1379-2407.

10. Ultimate theoretical strength of fcc $\mathrm{Fe}-\mathrm{Ni}$ alloy polycrystals / S. A. Starikov, A. R. Kuznetsov, L. E. Karkina, V. V. Sagaradze // Diagnostics, Resource and Mechanics of materials and structures. - 2015. - Iss. 6. - P. 58-62. - DOI: 10.17804/2410-9908.2015.6.058-062. URL: http://dream-journal.org. 
11. Sun Y., Rice J. R., Truskinovsky L. Dislocation Nucleation Versus Cleavage in $\mathrm{Ni}_{3} \mathrm{AI}$ and Ni // Mat. Res. Soc. Symp. Proc. - 1991. - Vol. 213. - P. 243-248. - DOI: 10.1557/PROC-213243.

12. Kelly A., Tyson W., Cottrell A. H. Ductile and brittle crystals // Phil. Mag. - 1967. Vol. 15, iss. 135. - P. 567-586. - DOI: 10.1080/14786436708220903.

13. Rosato V. Comparative behavior of carbon in bcc and fcc iron // Acta Metall. - 1989. Vol. 37, iss. 10. - P. 2759-2763. - DOI: 10.1016/0001-6160(89)90310-6.

14. Daw M. S., Baskes M. I. Embedded atom method: derivation and application to impurities, surfaces and other defects in metals // Phys. Rev. - 1984. - Vol. 29B, no. 12. - P. 6443-6453. DOI: 10.1103/PhysRevB.29.6443.

15. Johnson R. A., Dienes G. J., Damask A. C. Calculation of the energy and migration characteristics of carbon and nitrogen in $\alpha$-iron and vanadium // Acta Metall. - 1964. - Vol. 12, iss. 11. P. 1215-1224. - DOI: 10.1016/0001-6160(64)90105-1.

16. Molecular dynamics simulation and theoretical analysis of carbon diffusion in cementite / E. V. Levchenko, A. V. Evteev, I. V. Belova, G. E. Murch // Acta Mater. - 2009. - Vol. 57, iss. 3. P. 846-853. - DOI: 10.1016/j.actamat.2008.10.025.

17. Kar'kina L. E., Kar'kin I. N., Kuznetsov A. R Atomistic simulation of stacking faults in (001), (010), and (100) planes of cementite // Physics of Metals and Metallography. - 2014. Vol. 115, iss. 1. - P. 85-97. - DOI: 10.1134/S0031918X14010086.

18. Kar'kina L. E., Kar'kin I. N., Zubkova T. A. Atomistic simulation of stacking faults in cementite: Planes containing vector [100] // Physics of Metals and Metallography. - 2014. - Vol. 115, iss. 8. - P. 814-829. - DOI: 10.1134/S0031918X14080067.

19. Kar'kina L. E., Kar'kin I. N. Atomistic simulation of stacking faults in cementite: Planes containing vector [010] // Physics of Metals and Metallography. - 2014. - Vol. 115, iss. 8. P. 830-842. - DOI: 10.1134/S0031918X14080079.

20. Kar'kina L. E., Zubkova T. A., Yakovleva I. L. Dislocation structure of cementite in granular pearlite after cold plastic deformation // Physics of Metals and Metallography. - 2013. Vol. 114, iss. 3. - P. 234-241. - DOI: 10.1134/S0031918X13030095. 